ID

\title{
Surgical Treatment of Periimplantitis With Non-Augmentative Techniques
}

\author{
Philip L. Keeve, DMD, MS, ${ }^{\star} \dagger$ Ki Tae Koo, DDS, MS, PhD, $\ddagger$ Ausra Ramanauskaite, DDS, $\S$ \\ Georgios Romanos, DDS, PhD, ๆ Frank Schwarz, DDS, PhD, \| Anton Sculean, DMD, MS, PhD,\# \\ and Fouad Khoury, DMD, PhD*^††
}

$\mathrm{P}$ eriimplant diseases are defined as "collective term for inflammatory reactions in the tissues surrounding the implants," whereas periimplantitis was introduced as an inflammatory process on hard and soft tissue, resulting in pathological pocket formation and loss of supporting bone. ${ }^{2}$

The wide range in prevalence rates of $2.7 \%$ to $47.1 \%$ of implants ${ }^{1,3,4}$ can be attributed to differences in the study population, disease dentition, and implant micro- and macrostructures. Therefore, an effective strategy for treating this disease is required, otherwise a debilitating condition around the affected implants will result in loss of function and esthetics.

The development of an adherent biofilm on the implant surface plays an important role in the etiology of periimplantitis. ${ }^{2}$ As a result of this multifactorial, but significant role of bacteria in the initiation and progress of infection

*Private Practice, Hameln, Germany.

†Head, Department of Periodontology, Private Dental Clinic Schloss Schellenstein, Olsberg, Germany.

Professor, Department of Periodontology, Dental Research Institute, Seoul National University School of Dentistry, Seoul, Republic of Korea.

§Research Associate, Department of Oral Surgery,

.

qProfessor, Department of Periodontology, School of Dental

MPressor, Department of Periodontology, School of

Medicine, Stony Brook University, Stony Brook, NY.

Implantology, Carolinum, Johann Wolfgang Goethe-University

Frankfurt Frankfurt, Germany.

PProfessor Chairman, Department of Periodontology, School of Dental Medicine University of Bern, Bern, Switzerland.

** Director, Private

**Director, $P$.

††Professor, Department of Oral and Maxillo-Facial-Surgery,

University Muenster, Muenster, Germany.

Reprint requests and correspondence to: Philip $L$.

Keeve, DMD, MS, Suentelstrasse 10-12, 31785 Hameln,

Germany, Phone: +49515195760, Fax: +495151957611,

E-mail:pl.keeve@outlook.com

ISSN 1056-6163/18/02801-001

Implant Dentistry

Volume 28 - Number 1

Copyright $(2018$ Wolters Kluwer Health, Inc. All rights

reserved.

DOI: 10.1097/ID.0000000000000838
Objectives: The aim of this review was to systematically screen the literature on surgical non-regenerative treatments of periimplantitis, especially for radiologic and clinical outcomes, and to determine predictable therapeutic options for the clinical management of periimplantitis lesions.

Material and Methods: The potentially relevant literature was assessed independently by 2 reviewers to identify clinical studies, trials, and case series in humans describing the surgical non-regenerative treatment outcomes of periimplantitis with a follow-up of at least 6 months. MEDLINE, EMBASE, and the Cochrane Library were searched for studies reporting changes in probing depth (PD) and/or bleeding on probing (BOP) and/or radiologic marginal bone-level changes.

Results: A total of 10 publications were included: 6 prospective randomized controlled trials, 1 prospective cohort study, 2 retrospective controlled studies, and 1 case series. Clinical parameters can be reduced by surgical

of periimplant diseases, elimination of the established biofilm from the implant surface is the main objective in the treatment of periimplant mucositis and periimplantitis. ${ }^{5}$

Several clinical protocols for the treatment of periimplantitis have been proposed, including mechanical debridement, the use of antiseptics non-regenerative treatments. Concerning 3 year follow-ups, BOP and $P D$ values decreased more efficiently after implantoplasty than using systematic administration of antibacterials. Adjunctive local chemical irrigations or diode laser have no long-term effects. The non-regenerative surgical approach in combination with implantoplasty also shows improved radiographic parameters.

Conclusions: Surgical nonregenerative treatment of periimplantitis can reduce the amount of inflammation in the short-term follow-up. Using implantoplasty may result in the improvement of clinical and radiographic parameters. Because of limited evidence and heterogeneity in study design, there is a need for randomized controlled studies with proper design and powerful sample size in the future. (Implant Dent 2018;28:1-10)

Key Words: periimplant, dental implants, periodontitis, periimplant disease, CIST, resective, nonaugmentative, therapy, implantoplasty

and local or systemic antibiotics, ${ }^{6,7}$ surgical access, ${ }^{8-10}$ and regenerative $e^{11-14}$ or resective surgical procedures. ${ }^{15-18}$

The aim of this review is to systematically screen the literature on surgical non-regenerative treatments of periimplantitis, especially for radiologic and clinical outcomes, and to determine predictable therapeutic 
options for the clinical management of periimplantitis lesions.

\section{Materials and Methods}

A literature research was performed in MEDLINE via the PubMed database of the US National Library of Medicine, Ovid MEDLINE, EMBASE, and Dentistry and Oral Sciences Source for articles published between January 2005 and January 2018 using Medical Subject Heading search terms "periimplantitis" OR "peri-implantitis" OR "periimplant" OR "peri-implant" or "implant" AND "failure" AND "surgical" OR "treatment" OR "therapy" OR "non-regenerative" OR "nonregenerative" OR resective OR "laser" OR "lasers" OR “implantoplasty” OR "osteoplasty" OR "flap debridement" + free text terms, and in different combinations. To be included in the study, studies were screened by 2 independent reviewers and had to be

(i) written in the English language;

(ii) published in an international peerreviewed journal;

(iii) clinical studies or clinical trials in humans;

(iv) prospective or retrospective studies;

(v) case series.

Case reports, letters, editorials, and literature reviews were excluded.

According to disease definition, periimplantitis was defined as a clear radiographic threshold of more than $2 \mathrm{~mm}$ of marginal bone loss beyond biological periimplant bone remodeling, presence of bleeding on probing (BOP), and/or suppuration. ${ }^{19}$

Finally, the matching full-text articles were included if they fulfilled the following criteria:

- surgical non-regenerative/resective treatment in patients with at least 1 osseointegrated screw-shaped dental implant affected by "periimplantitis" mentioned above

- minimal sample size of 10 implants

- minimum 6 months of follow-up period

- description of at least 1 surgical non-regenerative treatment method of periimplantitis
- Report on clinical and radiographic periimplant tissue changes, including probing depth (PD) and/or BOP as the primary outcome measure and/or radiographic bone-level (RBL) change as the secondary outcome measure

The exclusion criteria were defined as follows:

- In vitro and in vivo studies

- Studies with inclusion of patients with severe systemic diseases and uncontrolled metabolic disorder or osteoporosis

- Studies concerning ceramic or coated implant surfaces

- Insufficient information for the review, including unavailability of authors

\section{Risk of Bias Assessment}

The following criteria were used according to the randomized clinical trial checklist of the Cochrane Center ${ }^{20}$ and the CONSORT statement. ${ }^{21}$ The degrees of bias were categorized as follows: low risk, if all the criteria were met; moderate risk, if 1 criterion was missing; and high risk, if 2 or more criteria were missing.

\section{Data Extraction and Method of Analysis}

All data from the eligible studies were extracted with a data extraction template. For data analysis, author and year of publication, type of study, sample size, antimicrobial adjunctive agents, detoxification methods, as well as PD change, BOP change, and RBL change before treatment and after respective healing periods including the duration of follow-up were extracted (Table 1).

\section{Results}

After initial screening of 863 potentially relevant publications because of data extraction and analysis described above, 724 articles were excluded and 139 studies were included, aiming on non-regenerative surgical treatments of periimplantitis. Among these 139 studies, furthermore 129 were excluded because of lack of information regarding the topic, the criteria for "diagnosis peri- implantitis" did not match, or the followup period was too short. Finally, 10 articles were included in this review. Six prospective randomized controlled trials, $, 9,10,16-18,221$ prospective cohort study, ${ }^{8} 2$ retrospective controlled studies, ${ }^{15,23}$ and 1 case series ${ }^{24}$ were designed with follow-up periods of 6 months, ,,24 1 year, ${ }^{8,10,15,22,23}$ and 3 years. ${ }^{16-18}$ The risk of bias across studies was classified as unclear risk in 5 cases ( 1 or more criteria missing), $8,9,17,18,22$ as moderate risk in 1 case, ${ }^{10}$ and as high risk in 4 studies ( 2 or more criteria missing). ${ }^{15,16,23,24}$

Clinical periimplant parameters as PD and BOP improved after access apical surgery only, ${ }^{8,15-17}$ surface decontamination with chlorhexidine (CHX), and/or cetylpyridinium chloride (CPC) $)^{15,23}$ and systemic antibiotics. ${ }^{8,18,22,24}$ One study showed no clinical benefits using adjunctive systemic azithromycin in combination with open flap debridement ${ }^{10}$; another study stated no potential benefits of systemic antibiotics over 3 years. ${ }^{18}$ The clinical parameters improved significantly, especially in studies performing implantoplasty ${ }^{17}$ or use of systemic antimicrobials. $^{8,22}$ In detail, implantoplasty (diamond/Arkansas burs + silicone polishers) as an adjunct to open flap debridement with bone recontouring and apical flap repositioning resulted in better BOP and PD scores, but higher mean mucosal recessions compared with the control group (PD: $1.64 \pm 1.29$ vs $2.3 \pm$ $1.45 \mathrm{~mm}$ ), where persistent active signs of periimplant inflammation recurred in all patients after 24 months. Adjunctive benefits, derived from the addition of resective surgical treatment consisting of apically repositioned flap, bone recontouring, and surface debridement with $0.12 \% \mathrm{CHX}+0.05 \% \mathrm{CPC}$ to a placebo solution (without CHX/CPC), ${ }^{15,23}$ tend to show greater immediate suppression of anaerobic bacteria on the implant surface than a placebo solution, but do not lead to superior clinical results or differences in mean marginal bone loss at 12 months of follow-up. Similarly, the adjunctive use of 980-nm diode laser to mechanical open flap debridement ${ }^{9}$ failed to reveal any significant clinical improvements in mean BOP and PD scores at the 6-month follow-up (Figs. 1-5).

Stable radiographic periimplant bone levels were observed after 


\begin{tabular}{|c|c|c|c|c|c|c|c|c|c|c|c|c|}
\hline Study & Year & Type of Study & Sample Size & $\begin{array}{c}\text { Follow-up } \\
(\mathrm{m})\end{array}$ & $\begin{array}{l}\text { Implant } \\
\text { Surface }\end{array}$ & $\begin{array}{l}\text { Perimplantitis } \\
\text { Definition }\end{array}$ & $\begin{array}{c}\text { Treatment Method } \\
\text { Used }\end{array}$ & $\begin{array}{l}\text { Decontamination } \\
\text { Method }\end{array}$ & $\begin{array}{l}\text { Outcomes } \\
\text { Evaluated }\end{array}$ & $\begin{array}{l}\text { Mean PD Changes } \\
\text { (SD) }\end{array}$ & $\begin{array}{c}\text { Mean BOP } \\
\text { Changes Mean } \\
\text { (SD) }\end{array}$ & $\begin{array}{c}\text { Radiographic } \\
\text { Marginal Bone-Level } \\
\text { Changes }\end{array}$ \\
\hline $\begin{array}{l}\text { Heitz-Mayfield } \\
\text { et } \mathrm{al}^{8}\end{array}$ & 2012 & $\begin{array}{l}\text { Prospective } \\
\text { cohort } \\
\text { study }\end{array}$ & $\begin{array}{l}24 \text { patients; } \\
\text { mean age } 56 \\
\text { years }\end{array}$ & 12 & $\begin{array}{l}36 \text { implants, } \\
\text { rough }\end{array}$ & $\begin{array}{l}\text { BOP and/or pus } \\
\text { on probing }+ \\
\mathrm{PD} \geq 5 \mathrm{~mm} \\
\text { and bone loss } \\
\geq 2 \mathrm{~mm}\end{array}$ & $\begin{array}{l}\text { Open flap } \\
\text { debridement and } \\
\text { implant surface } \\
\text { decontamination } \\
\text { with saline and } \\
\text { with adjunctive } \\
\text { systemic } \\
\text { amoxiclilin and } \\
\text { metronidazole }\end{array}$ & Sterile saline & $\begin{array}{l}\text { Clinical: PD, } \\
\text { recessions, } \\
\text { BOP, and } \\
\text { pus; } \\
\text { radiographic: } \\
\text { MBL changes }\end{array}$ & 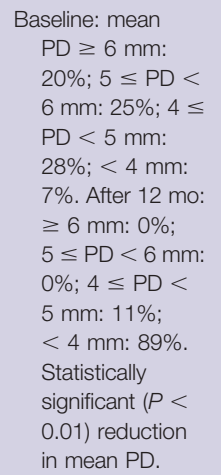 & $\begin{array}{l}\text { Number of sites } \\
\text { with BOP: } \\
\text { baseline: } 2.5 \\
\text { (1); after } 12 \\
\text { mo: } 1 \text { (1.2) } \\
\text { Statistically } \\
\text { signicant ( } P< \\
\text { 0.01) } \\
\text { reduction in } \\
\text { BOP. }\end{array}$ & $\begin{array}{l}\text { Three implants in } \\
3 \text { patients had } \\
0.6-1 \mathrm{~mm} \text { bone } \\
\text { loss at } 12 \mathrm{mo} \text {. } \\
\text { Three implants in } \\
3 \text { patients } \\
\text { showed bone } \\
\text { gain, whereas } \\
\text { the remaining } \\
\text { implants had } \\
\text { stable marginal } \\
\text { bone levels. }\end{array}$ \\
\hline $\begin{array}{l}\text { Papadopoulos } \\
\text { et a } a^{9}\end{array}$ & 2015 & $\begin{array}{l}\text { Randomized } \\
\text { controlled } \\
\text { clinical } \\
\text { study }\end{array}$ & $\begin{array}{l}\text { 16: age } 55 \text { years. } \\
\text { (40-73) group } \\
\text { 1: } 8 \text {, group 2: } \\
8\end{array}$ & 6 & Not known & $\begin{array}{l}\text { BOP and/or pus } \\
\text { on probing }+ \\
\text { PD } \geq 6 \mathrm{~mm} \\
\text { and bone } \\
\text { loss } \geq 2 \mathrm{~mm}\end{array}$ & $\begin{array}{l}\text { Group 2: open flap } \\
\text { debridement with } \\
\text { the additional } \\
\text { use of diode } \\
\text { laser }\end{array}$ & $\begin{array}{l}\text { Group 1: use of } \\
\text { cotton swabs } \\
\text { soaked in saline } \\
\text { solution } \\
\text { Group 2: saline } \\
\text { solution + diode } \\
\text { laser for } 2 \text { min, } \\
532 \mathrm{~nm} \text {; }\end{array}$ & $\begin{array}{l}\text { Clinical: PD, } \\
\text { recessions, } \\
\text { BOP, pus, } \\
\text { and plaque } \\
\text { index; } \\
\text { radiographic: } \\
\text { MBL changes }\end{array}$ & $\begin{array}{l}\text { Group 1: baseline } \\
\text { mean PD } \\
5.92 \mathrm{~mm} \text {; after } 6 \\
\text { mo } 4.44 \mathrm{~mm} \text {; } \\
\text { reduction of } \\
\text { 1.38 mm. } \\
\text { Group 2: baseline } \\
\text { mean PD } \\
5.52 \mathrm{~mm} \text {; after } 6 \\
\text { mo } 4.31 \mathrm{~mm} \text {; } \\
\text { reduction of } \\
1.19 \mathrm{~mm} \text {. } \\
\\
\text { No statistically } \\
\text { significant } \\
\text { difference } \\
\text { between the } 2 \\
\text { groups. }\end{array}$ & $\begin{array}{l}\text { Group 1: baseline } \\
\text { 93.5\%; after } 6 \\
\text { mo } 31.3 \% ; \\
\text { mean } \\
\text { reduction } \\
\text { 72.9\%. } \\
\text { Group 2: baseline } \\
\text { 81.2\%; after } 6 \\
\text { mo } 23.8 \% ; \\
\text { mean } \\
\text { reduction } \\
66.7 \% \\
(P<0.05) \\
\text { No statistically } \\
\text { significant } \\
\text { difference } \\
\text { between } \\
\text { groups }\end{array}$ & \\
\hline \multirow[t]{2}{*}{$\begin{array}{l}\text { de Waal } \\
\text { et al }{ }^{15}\end{array}$} & 2013 & $\begin{array}{l}\text { Retrospective } \\
\text { clinical } \\
\text { study }\end{array}$ & $\begin{array}{l}\text { 30: group } 1: 15 \\
\text { age } 61.5 \\
\text { years; group } \\
\text { 2: } 15 \text {, age } \\
59.4 \text { years }\end{array}$ & 12 & $\begin{array}{l}79 \text { implants, } \\
\text { rough }\end{array}$ & $\begin{array}{l}\text { BOP and/or pus } \\
\text { on probing }+ \\
\mathrm{PD} \geq 5 \mathrm{~mm} \\
\text { and bone loss } \\
\geq 2 \mathrm{~mm}\end{array}$ & $\begin{array}{l}\text { Resective surgery } \\
\text { with bone } \\
\text { recontouring and } \\
\text { surface } \\
\text { decontamination. }\end{array}$ & $\begin{array}{l}\text { Test: } 0.12 \% \\
\text { CHX+0.05\% } \\
\text { cetylpyridinium } \\
\text { chloride (CPC); } \\
\text { control: no } \\
\text { CHXICPC }\end{array}$ & $\begin{array}{l}\text { Clinical: BOP, } \\
\text { PD, and pus; } \\
\text { radiographic: } \\
\text { MBL loss } \\
\text { after } 1 \text { y and } \\
\text { comparison } \\
\text { between the } \\
\text { groups }\end{array}$ & $\begin{array}{l}\text { Mean PD } \geq 5 \mathrm{~mm} \text { : } \\
\text { group 1: } \\
\text { baseline } 88.2 \\
(18.4) \% \text {; after } \\
12 \mathrm{mo} 733.9 \\
(39) \% .\end{array}$ & $\begin{array}{l}\text { Group 1: baseline } \\
87.1(27) \% \text {; } \\
\text { after } 12 \mathrm{mo} \\
25.8(8) \% .\end{array}$ & \multirow[t]{2}{*}{$\begin{array}{l}\text { Group 1: baseline } \\
\text { 4.3 (2.1) } \mathrm{mm} \text {; } \\
\text { after } 12 \mathrm{mo} 5 \\
\text { (2.5) } \mathrm{mm} \text {. } \\
\text { Group 2: } \\
\text { baseline } 3.61 \\
\text { (1.9) } \mathrm{mm} \text {; after } \\
12 \mathrm{mo} 3.9 \\
\text { (2) } \mathrm{mm} .\end{array}$} \\
\hline & & & & & & & $\begin{array}{l}\text { Group 1: } 0.12 \% \\
\text { CHX }+0.05 \% \\
\text { CPC. }\end{array}$ & & & $\begin{array}{l}\text { Group 2: baseline } \\
\quad 75.2(26.1) \% ; \\
\text { after } 12 \mathrm{mo} 17.1 \\
\text { (24)\% }\end{array}$ & $\begin{array}{l}\text { Group 2: baseline } \\
\text { 81.3 (39)\%; } \\
\text { after } 12 \text { mo } \\
15.8(6) \% .\end{array}$ & \\
\hline
\end{tabular}

(continued on next page) 


\begin{tabular}{|c|c|c|c|c|c|c|c|c|c|c|c|c|}
\hline Study & Year & Type of Study & Sample Size & $\begin{array}{l}\text { Follow-up } \\
(\mathrm{m})\end{array}$ & $\begin{array}{l}\text { Implant } \\
\text { Surface }\end{array}$ & $\begin{array}{l}\text { Perimplantitis } \\
\text { Definition }\end{array}$ & $\begin{array}{c}\text { Treatment Method } \\
\text { Used }\end{array}$ & $\begin{array}{l}\text { Decontamination } \\
\text { Method }\end{array}$ & $\begin{array}{l}\text { Outcomes } \\
\text { Evaluated }\end{array}$ & $\begin{array}{l}\text { Mean PD Changes } \\
\text { (SD) }\end{array}$ & $\begin{array}{l}\text { Mean BOP } \\
\text { Changes Mean } \\
\text { (SD) }\end{array}$ & $\begin{array}{c}\text { Radiographic } \\
\text { Marginal Bone-Level } \\
\text { Changes }\end{array}$ \\
\hline \multirow{3}{*}{ Romeo et al ${ }^{17}$} & \multirow{3}{*}{2005} & \multirow{3}{*}{$\begin{array}{l}\text { Randomized } \\
\text { clinical } \\
\text { study }\end{array}$} & \multirow{3}{*}{$\begin{array}{l}\text { 17: group 1: 10; } \\
\text { group 2: } 7\end{array}$} & \multirow{3}{*}{36} & \multirow{3}{*}{$\begin{array}{c}35 \text { implants, } \\
\text { rough }\end{array}$} & \multirow{3}{*}{$\begin{array}{l}\text { Pus or BOP, PD > } \\
4 \text { mm, no } \\
\text { mobility, and } \\
\text { radiographic } \\
\text { horizontal bone } \\
\text { loss }\end{array}$} & Group 2: placebo. & & & $\begin{array}{l}\text { Mean PD } \geq 6 \text { mm: } \\
\text { Group 1: baseline } \\
54.5(33.7) \% ; \\
\text { after 12 mo } 17.7 \\
\text { (34.3)\%. } \\
\text { Group 2: baseline } \\
\text { 46.9 (33.7)\%; } \\
\text { after } 12 \text { mo } 17.2 \\
\text { (19.2)\%. }\end{array}$ & & \\
\hline & & & & & & & $\begin{array}{l}\text { Group } 1 \text { (test): } \\
\text { resective surgery } \\
\text { and modification } \\
\text { of surface } \\
\text { topography } \\
\text { (implantoplasty) }\end{array}$ & \multirow[t]{2}{*}{$\begin{array}{l}\text { Metronidazole + } \\
\text { tetracycline } \\
\text { hydrochloride } \\
\text { (3 min) }\end{array}$} & \multirow[t]{2}{*}{$\begin{array}{l}\text { Clinical: survival } \\
\text { rate, PD, mBl, } \\
\text { and mucosal } \\
\text { recession } \\
\text { index }\end{array}$} & $\begin{array}{l}\text { Group 1: baseline } \\
5.79(1.69) \mathrm{mm} \\
\text { after } 36 \mathrm{mo} 3.21 \\
\text { (0.56) mm }\end{array}$ & $\begin{array}{l}\text { Group 1: baseline } \\
\text { 2.83 (0.47); } \\
\text { after } 36 \mathrm{mo} \\
0.61 \text { (0.67) }\end{array}$ & \\
\hline & & & & & & & $\begin{array}{l}\text { Group 2: resective } \\
\text { surgery only } \\
\text { (control group) }\end{array}$ & & & $\begin{array}{l}\text { Group 2: baseline } \\
6.52(1.62) \mathrm{mm} \text {; } \\
\text { after } 24 \mathrm{mo} 5.5 \\
(1.47) \mathrm{mm} .\end{array}$ & $\begin{array}{c}\text { Group 2: baseline } \\
2.86(0.35) ; \\
\text { after } 24 \text { mo } \\
2.33(0.75)\end{array}$ & \\
\hline Romeo et al16 & 2007 & $\begin{array}{l}\text { Randomized } \\
\text { clinical } \\
\text { study }\end{array}$ & $\begin{array}{l}\text { 19: group 1: 10; } \\
\text { group 2: } 9\end{array}$ & 36 & $\begin{array}{l}38 \text { implants, } \\
\text { rough }\end{array}$ & $\begin{array}{l}\text { Pus or BOP, PD } \\
>4 \mathrm{~mm} \text {, no } \\
\text { mobility, } \\
\text { radiographic } \\
\text { horizontal } \\
\text { bone loss }\end{array}$ & $\begin{array}{l}\text { Group } 1 \text { (test): } \\
\text { resective surgery } \\
\text { and } \\
\text { implantoplasty. } \\
\text { Group } 2 \\
\text { (control): } \\
\text { resective surgery } \\
\text { alone. }\end{array}$ & $\begin{array}{l}\text { Metronidazole + } \\
\text { tetracycline } \\
\text { hydrochloride } \\
\text { (3 min) }\end{array}$ & $\begin{array}{l}\text { Radiographic: } \\
\text { marginal } \\
\text { bone loss }\end{array}$ & & & $\begin{array}{l}\text { Group 1: baseline } \\
\text { mesially } 3.82 \\
\text { (1.52) mm, } \\
\text { distally } 3.94 \\
\text { (1.64) mm; after } \\
3 \text { y mesially } \\
3.81(3.94) \mathrm{mm}, \\
\text { distally } 1.72 \\
\text { (1.79) mm. } \\
\text { Group } 2: \text { baseline } \\
\text { mesially } 3.45 \\
\text { (1.93) } \mathrm{mm}, \\
\text { distally } 3.49 \\
(1.8) \mathrm{mm} \text {; after } \\
3 \text { y mesially } \\
5.35(1.99) \mathrm{mm}, \\
\text { distally } 5.42 \\
\text { (1.91) } \mathrm{mm} \\
\text { Significantly higher } \\
(P<0.05) \\
\text { mean MBL was } \\
\text { recorded in } \\
\text { group } 2 \text { than in } \\
\text { group } 1 .\end{array}$ \\
\hline $\begin{array}{l}\text { de Waal } \\
\text { et al23 }\end{array}$ & 2015 & $\begin{array}{l}\text { Retrospective } \\
\text { clinical } \\
\text { study }\end{array}$ & $\begin{array}{l}\text { 44: group } 1: 22 \text {, } \\
\text { age } 60.5 \\
\text { years; group } \\
\text { 2: } 22, \text { age } \\
58.6 \text { years }\end{array}$ & 12 & $\begin{array}{l}108 \\
\text { implants, } \\
\text { rough }\end{array}$ & $\begin{array}{l}\text { BOP and/or pus } \\
\text { on probing }+ \\
\text { PD } \geq 5 \mathrm{~mm} \\
\text { and bone loss } \\
\geq 2 \mathrm{~mm}\end{array}$ & $\begin{array}{l}\text { Resective surgery } \\
\text { with bone } \\
\text { recontouring and } \\
\text { surface } \\
\text { decontamination. }\end{array}$ & $\begin{array}{l}\text { Test: } 2.0 \% \mathrm{CHX} \\
\text { control: } 0.12 \% \\
\text { CHX+0.05\% CPC }\end{array}$ & $\begin{array}{l}\text { Clinical: BOP, } \\
\text { PD, and pus; } \\
\text { radiographic: } \\
\text { MBL loss } \\
\text { after } 1 \text { y and } \\
\text { comparison } \\
\text { between the } \\
\text { groups }\end{array}$ & $\begin{array}{l}\text { Mean PD } \geq 5 \mathrm{~mm} \text { : } \\
\text { Group 1: baseline } \\
57.5 \text { (26.6)\%; } \\
\text { after } 12 \mathrm{mo} 7.3 \\
\text { (12.6)\%. }\end{array}$ & $\begin{array}{c}\text { Group 1: baseline } \\
\text { 82.1 (23.9)\%; } \\
\text { after } 12 \mathrm{mo} \\
42.7(34.2) \%\end{array}$ & $\begin{array}{l}\text { Group 1: baseline } \\
4(1.5) \mathrm{mm} \text {; } \\
\text { after } 12 \mathrm{mo} 4.3 \\
\text { (1.7) } \mathrm{mm} \text {. }\end{array}$ \\
\hline
\end{tabular}




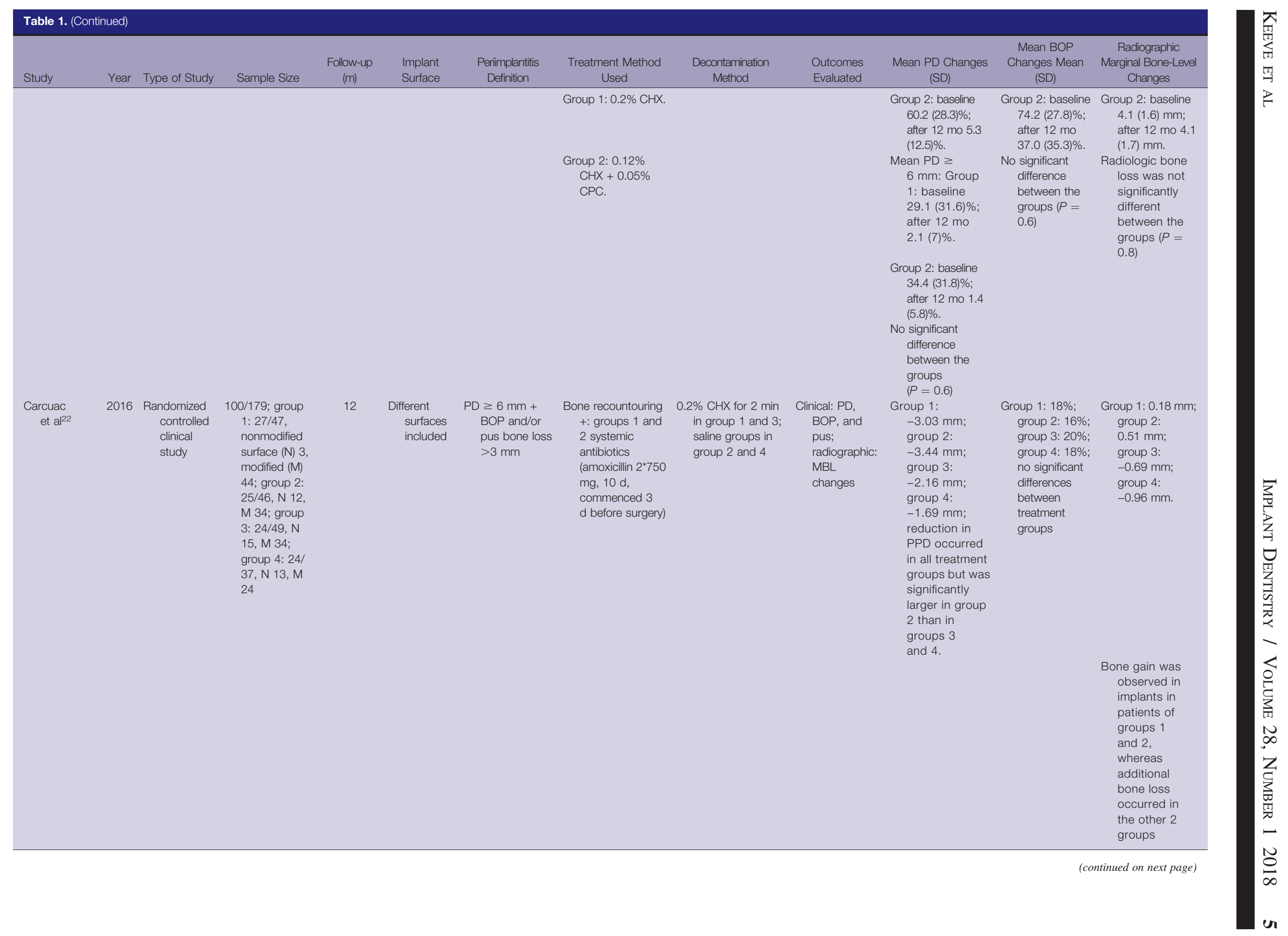




\begin{tabular}{|c|c|c|c|c|c|c|c|c|c|c|c|c|}
\hline Study & Year & Type of Study & Sample Size & $\begin{array}{l}\text { Follow-up } \\
(m)\end{array}$ & $\begin{array}{l}\text { Implant } \\
\text { Surface }\end{array}$ & $\begin{array}{l}\text { Perimplantitis } \\
\text { Definition }\end{array}$ & $\begin{array}{c}\text { Treatment Method } \\
\text { Used }\end{array}$ & $\begin{array}{l}\text { Decontamination } \\
\text { Method }\end{array}$ & $\begin{array}{l}\text { Outcomes } \\
\text { Evaluated }\end{array}$ & $\begin{array}{l}\text { Mean PD Changes } \\
\text { (SD) }\end{array}$ & $\begin{array}{l}\text { Mean BOP } \\
\text { Changes Mean } \\
\text { (SD) }\end{array}$ & $\begin{array}{c}\text { Radiographic } \\
\text { Marginal Bone-Level } \\
\text { Changes }\end{array}$ \\
\hline & & & & & & & & & & & & $\begin{array}{l}>P<0.05 \\
\text { groups } 1 \text { and } 2 \\
\text { vs groups } 3 \\
\text { and } 4\end{array}$ \\
\hline $\begin{array}{l}\text { Carcuac } \\
\text { et al }\left.\right|^{18}\end{array}$ & 2017 & $\begin{array}{l}\text { Randomized } \\
\text { controlled } \\
\text { clinical } \\
\text { study }\end{array}$ & $\begin{array}{l}\text { 67/121 group 1: } \\
68 \text { implants; } \\
\text { group 2: } \\
53 \text { implants; } \\
\text { group 3: } \\
90 \text { implants; } \\
\text { group 4: } \\
31 \text { implants }\end{array}$ & 36 & $\begin{array}{l}\text { Different } \\
\text { surfaces } \\
\text { included }\end{array}$ & $\begin{array}{l}\mathrm{PD} \geq 6 \mathrm{~mm}+ \\
\text { BOP and/or } \\
\text { pus bone loss } \\
>3 \mathrm{~mm}\end{array}$ & $\begin{array}{l}\text { Bone recountouring } \\
\text { +: Groups } 1 \text { and } \\
2 \text { systemic } \\
\text { antibiotics } \\
\text { (amoxicillin } 2^{\star} 750 \\
\mathrm{mg}, 10 \mathrm{~d}, \\
\text { commenced } 3 \mathrm{~d} \\
\text { before surgery) }\end{array}$ & $\begin{array}{l}0.2 \% \mathrm{CHX} \text { for } 2 \text { min } \\
\text { in group } 1 \text { and } 3 \text {; } \\
\text { saline groups in } \\
\text { group } 2 \text { and } 4\end{array}$ & $\begin{array}{l}\text { Clinical: PD, } \\
\text { BOP, and } \\
\text { pus; } \\
\text { radiographic: } \\
\text { MBL } \\
\text { changes }\end{array}$ & $\begin{array}{l}\text { Group 1: -3 mm; } \\
\text { group 2: } \\
\text {-2.38 mm; } \\
\text { group 3: } \\
\text {-2.67 mm; } \\
\text { group 4: } \\
\text {-2.9 mm; PD } \\
\text { reduction was } \\
\text { more } \\
\text { pronounced at } \\
\text { the non- } \\
\text { modified sur- } \\
\text { face implants } \\
\text { and that } \\
\text { adjunctive use } \\
\text { of systemic an- } \\
\text { tibiotics } \\
\text { improved the } \\
\text { outcome at im- } \\
\text { plants with } \\
\text { modified surfa- } \\
\text { ces }\end{array}$ & $\begin{array}{l}\text { BoP/SoP+ was } \\
\text { lower for } \\
\text { implants with } \\
\text { non-modified } \\
\text { surfaces, } \\
\text { ranging from } \\
27 \% \text { to } 44 \% \text {, } \\
\text { when com- } \\
\text { pared with } \\
\text { modified sur- } \\
\text { face implants } \\
(70 \%) \text { Sys- } \\
\text { temic anti- } \\
\text { biotics had no } \\
\text { effect in terms } \\
\text { of BoP/SoP. }\end{array}$ & $\begin{array}{l}\text { Group 1: } \\
\text {-0.32 mm; } \\
\text { group 2: } \\
0.51 \mathrm{~mm} ; \\
\text { group 3: } \\
0.28 \mathrm{~mm} ; \\
\text { group 4: } \\
-0.65 \mathrm{~mm} .\end{array}$ \\
\hline $\begin{array}{l}\text { Hallström } \\
\text { et al }^{10}\end{array}$ & 2017 & $\begin{array}{l}\text { Randomized } \\
\text { controlled } \\
\text { clinical } \\
\text { study }\end{array}$ & $\begin{array}{c}\text { Control: 16/16; } \\
\text { test: 15/15 }\end{array}$ & 12 & $\begin{array}{l}\text { Different } \\
\text { surfaces } \\
\text { included, } \\
\text { rough }\end{array}$ & $\begin{array}{l}\text { BOP and/or pus } \\
\text { on probing }+ \\
\mathrm{PD} \geq 5 \mathrm{~mm} \\
\text { and bone loss } \\
\geq 2 \mathrm{~mm}\end{array}$ & $\begin{array}{l}\text { Test group: open } \\
\text { flap } \\
\text { debridement, } \\
\text { cleaning with } \\
\text { sterile curettes } \\
\text { and saline- } \\
\text { soaked cotton } \\
\text { gauzes, } \\
\text { Zithromax } \\
250 \mathrm{mg} \times 2 \text { on } \\
\text { the day of } \\
\text { surgery, and } \\
250 \mathrm{mg} \times 1 \text { per } \\
\text { day during } \\
4 \text { additional d; } \\
\text { control group: } \\
\text { Open flap } \\
\text { debridement, } \\
\text { cleaning with } \\
\text { sterile curettes } \\
\text { and saline- } \\
\text { soaked cotton } \\
\text { gauzes }\end{array}$ & $\begin{array}{l}\text { saline-soaked cotton } \\
\text { gauzes }\end{array}$ & $\begin{array}{l}\text { Clinical: PD, } \\
\text { BOP, and } \\
\text { pus; } \\
\text { radiographic: } \\
\text { MBL } \\
\text { changes; } \\
\text { microbial } \\
\text { samples }\end{array}$ & $\begin{array}{l}\text { Successful clinical } \\
\text { outcome: PPD } \\
\leq 5 \mathrm{~mm}, \text { no } \\
\text { suppuration, no } \\
\text { BOP at the } \\
\text { implant sites, } \\
\text { and bone loss } \\
\leq 0.5 \mathrm{~mm} \text {. } \\
\text { Based on the both } \\
\text { treatment } \\
\text { groups, } 11 / 31 \\
\text { individuals } \\
\text { (35.5\%) } \\
\text { presented with } \\
\text { a successful } \\
\text { treatment } \\
\text { outcome. }\end{array}$ & $\begin{array}{l}\text { Statistical analysis } \\
\text { failed to } \\
\text { demonstrate } \\
\text { differences in } \\
\text { BOP scores. }\end{array}$ & $\begin{array}{l}\text { Mean gain of } \\
\text { alveolar bone at } \\
\text { implants } \\
\text { assessed from } \\
\text { radiographs } \\
\text { was also } \\
\text { significant } \\
\text { (mean diff: } \\
0.4 \mathrm{~mm}, S E \\
\text { mean diff: } \\
0.2 \mathrm{~mm}, 95 \% \\
\text { Cl: } 0.0,0.8, P< \\
0.05 \text { ) }\end{array}$ \\
\hline
\end{tabular}




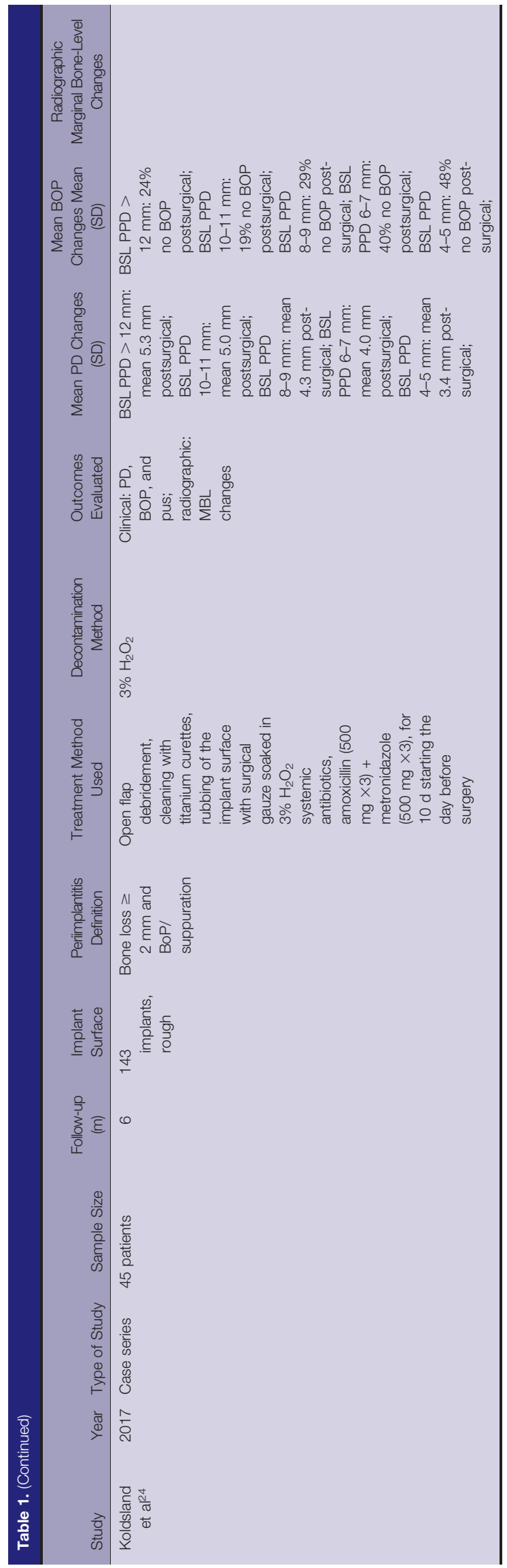

systemic application of antibiotics $8,10,22$ or local use of chemical compounds. ${ }^{15,23}$ In studies with significant improvement of clinical periimplant parameters after implantoplasty, RBLs and marginal bone loss were significantly lower after 3 years of followup, ${ }^{16}$ with interproximal bone loss at control sites up to 1.45 to $1.54 \mathrm{~mm}$.

A total number of 35 implants were removed after non-augmentative surgery and progressive recurrence of periimplant inflammation. ${ }^{15-18,22} \mathrm{Be}-$ cause of implant neck fracture, 1 implant was removed. ${ }^{23}$

Four studies reported on the smoking status of the patients, ranging from $25 \%{ }^{8}$ to $59.1 \%^{23}$ Despite 2 studies $^{8,10}$ reporting no negative effect of smoking on treatment outcome, smoking was influencing treatment success when adjusted for baseline and follow-up. ${ }^{15,23}$

\section{Discussion}

Surgical techniques are used depending on multiple factors including patient general health condition, oral hygiene, type of bony defects, implant surface, postoperative maintenance program, and other factors that cannot be completely assumed in a systematic literature review. The non-augmentative treatment concept is indicated for supracrestal bone defects (horizontal bone loss) with exposed threads in esthetically non-demanding areas based on patient needs and satisfaction and involves reduction or elimination of pathological periimplant pockets, the apical positioning of a mucosal flap, or recontouring bone with or without implant surface modification, called implantoplasty. In case of modifications of implant surfaces, the rough design should be removed and polished. However, a concern of remaining titanium particles should be addressed.

Even in cases of periimplant mucositis, it can be advisable to perform an access flap for proper mechanical and chemical decontamination (ie, additional removal of cement remnants) of the implant surface.

The resolution of infection can be achieved by a proper method of implant surface decontamination. Clinical studies comparing different decontamination 


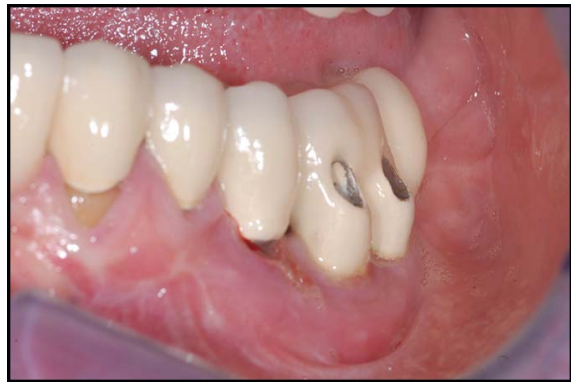

Fig. 1. Clinical situation (baseline) of a 53year-old patient suffering from periimplantitis in the left lower jaw, with massive soft-tissue swelling, redness, ulceration, and BOP. Implants were inserted 14 years ago, but there was no adherence to a supportive periodontal treatment for the past 6 years. Nevertheless, his oral hygiene was acceptable and implants were placed too buccally, but surrounded by adequate keratinized mucosa.

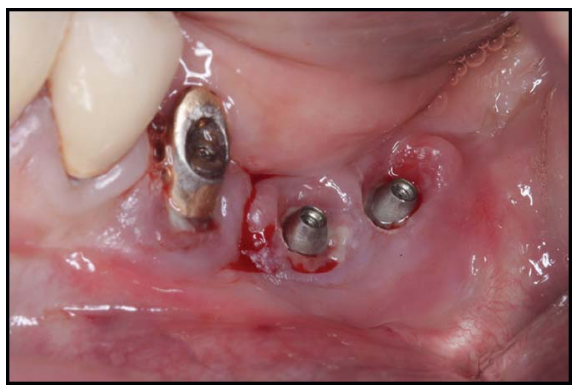

Fig. 2. Eight weeks after nonsurgical treatment with mechanical (plastic ultrasonic device) and chemical debridement (hydrogen peroxide 3\% and chlorhexidine digluconate solution $0.2 \%$, as well as adjunctive local antibiotics (tetracycline derivate), the clinical situation showed furthermore signs of massive inflammation. For a proper surgical treatment, including implantoplasty, it was absolutely necessary to remove the suprastructure, especially in the lower jaw.

methods or placebo failed to reveal significant differences between different, locally applied decontamination methods on clinical treatment outcomes. ${ }^{15,23} \mathrm{Nev}$ ertheless, a combined mechanical and chemical removal of biofilm is further recommended. ${ }^{5}$ Furthermore, adjunctive systemic antibiotics had no impact on the treatment's success for implants with a machined surface, and a positive effect was observed for the treatment success of implants with rough surfaces only in the first year. ${ }^{22}$ Local antibiotic delivery in addition to mechanical debridement and irrigation with an antimicrobial agent

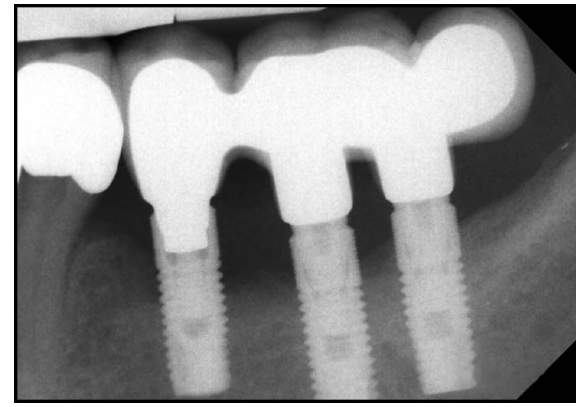

Fig. 3. Baseline radiographs showing horizontal bone loss with a supracrestal defect height of 3 to $5 \mathrm{~mm}$. In addition, vertical defects could be detected around the implants 35 and 36 with a depth of $3 \mathrm{~mm}$. The combination of the clinical and radiographic diagnostics stated a clear indication for surgical, non-augmentative treatment of the periimplantitis.

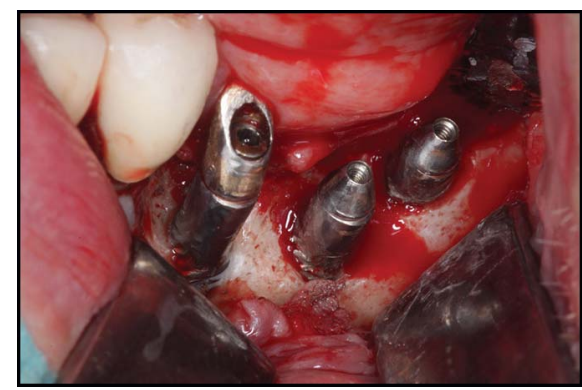

Fig. 4. A surgical, non-augmentative treatment of periimplantitis was performed, including an open flap debridement, after implantoplasty of infected and exposed implant surfaces, chemical surface decontamination and disinfection of the implants, and minimally invasive osteoplasty concerning only small intrabony defects. After minimal-invasive access flap, implant surfaces were treated with diamond burs and finally polished with greenies and supergreenies. Internal gingivectomy was completed before minimal-invasive osteoplasty and apical reposition flap with periosteal sutures.

may be an effective option for treating periimplantitis lesions. ${ }^{6,7}$ Moreover, the development of bacterial resistance seems to be an unlikely even in the event of repeated applications. ${ }^{25}$

Resective periimplantitis therapy combined with implantoplasty leads to superior clinical and radiographic treatment outcomes compared with resective treatment alone. In particular, implantoplasty, which can be performed with diamond or carbide burs and metal polishing instruments with

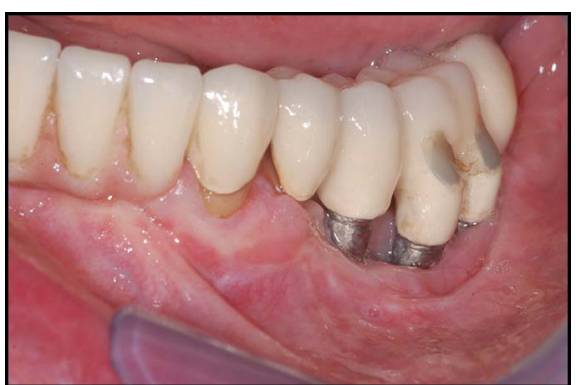

Fig. 5. After a 3-year-follow-up, no signs of inflammation, proper oral hygiene, and sufficient keratinized mucosa were present. Implant recessions of 2 to $3 \mathrm{~mm}$ resulted from the non-augmentative surgical periimplantitis. Supportive periodontal treatment took place every 3 months after the diagnosis of periimplantitis.

irrigation, positively influenced implant survival rates ( $100 \%$ test, $87.5 \%$ control group), significantly reduced periimplant pocket depths, and reduced bleeding scores. $^{17}$ Implantoplasty was associated with stabile interproximal bone levels at the 3-year follow-up, although significantly radiographic bone loss at control sites was detected. ${ }^{16}$ However, the gingival recession index was significantly higher when implant surface modification was performed. ${ }^{17}$ Therefore, this type of surgical technique can be of benefit primarily in the non-esthetic areas, and alternative treatments should be developed for the esthetic zone. ${ }^{5}$

The resective therapy outcomes were influenced by the experience of the surgical team, the amount of periimplant bone loss, maximum probing pocket depth at baseline, as well as a patient's smoking habits, and the presence of plaque during the follow-up. ${ }^{23}$

Analysis of studies and achieved reliability of treatments that revealed similarities in treatment approaches between protocols included (1) pretreatment phase, (2) cause-related therapy, and (3) maintenance phase. Oral hygiene instructions and its importance must be stressed to patients before and after the treatment. ${ }^{26}$ Nonsurgical subgingival mechanical debridement in conjunction with local antibacterials, such as chlorhexidine digluconate or locally delivered antibiotics, is effective in reducing soft-tissue inflammation ${ }^{7,27}$ and should be the first step in successful 
treatment. ${ }^{26}$ The supportive treatment phase is mandatory for the success of periimplantitis treatment. ${ }^{26}$ In a 5-year follow-up observational study, healthy periimplant tissue conditions in patients with high oral hygiene standard (maintenance every 6 months) could be maintained for most patients after resective periimplant surgery. ${ }^{28}$ Further disease progression occurred only in $10 \%$ of the treated implants, revealing that risk factors including the presence of residual pockets after surgery, smoking, poor oral hygiene, untreated periodontal disease, and diabetes may modify both the initial and long-term outcome of the treatment. ${ }^{26}$

All studies included implants with both cemented or screw-retained suprastructures. Hence, some surgeries were performed with suprastructures in place and some after removal of the suprastructures. All clinical assessments were performed with suprastructures in place. The suprastructure probably might affect quality of implant decontamination and modification and flap design and possibly the measurement of clinical parameters. If possible, it is strongly recommended to remove the suprastructure during surgical intervention and might be adapted for better cleansing ability.

Limitations of the revised studies are relatively small sample sizes and short follow-up periods. Furthermore, there were inconsistencies in methodology with various treatment modalities, limited control groups, different implant systems, and follow-up results from the same research groups. Unclear or high risk of bias of included studies may have an effect on the conclusions. ${ }^{20}$ The evidence of the review is limited due to significant variations observed in the included studies. Therefore, there is a substantial need for randomized controlled studies with a proper design and powerful sample size to provide strong and comparable evidence of benefits of non-augmentative procedures for treating periimplantitis.

\section{Conclusions}

The present systematic review showed that surgical non-regenerative modalities treating periimplantitis can reduce the amount of inflammation in the short-term follow-up, but seem less effective in the long-term perspective. Using implantoplasty in surgical nonregenerative treatment leads to a significant decrease in BOP and PD and may result in improvement of clinical and radiographic parameters up to 3 years after surgery compared with mechanical debridement alone. Application of systemic antibiotics, chemical compounds, or diode laser did not result in significant clinical or radiographic long-term improvements.

\section{Disclosure}

The authors claim to have no financial interest, either directly or indirectly, in the products or information listed in the manuscript.

\section{Roles/Contributions BY Authors}

P. L. Keeve: main author, corresponding author, and involved in writing/editing the manuscript. K. T. Koo: input to discussion. A. Ramanauskaite: input to material \& methods and editing the manuscript. G. Romanos: input to discussion. F. Schwarz: proofreading and revision of the manuscript. A. Sculean: input to introduction and results. F. Khoury: main proofreading and leading input to the manuscript.

\section{REFERENCES}

1. Zitzmann NU, Berglundh T. Definition and prevalence of peri-implant diseases. J Clin Periodontol. 2008;35:286-291.

2. Lang NP, Berglundh T. Periimplant diseases: Where are we now?-Consensus of the seventh European Workshop on Periodontology. J Clin Periodontol. 2011;38: 178-181.

3. Mir-Mari J, Mir-Orfila P, Figueiredo $R$, et al. Prevalence of peri-implant diseases. A cross-sectional study based on a private practice environment. J Clin Periodontol. 2012;39:490-494.

4. Koldsland OC, Scheie AA, Aass AM. Prevalence of peri-implantitis related to severity of the disease with different degrees of bone loss. J Periodontol. 2010; 81:231-238.

5. Renvert S, Polyzois IN. Clinical approaches to treat peri-implant mucositis and peri-implantitis. Periodontol 2000. 2015;68:369-404.
6. Mombelli A, Feloutzis A, Bragger U, et al. Treatment of peri-implantitis by local delivery of tetracycline. Clinical, microbiological and radiological results. Clin Oral Implants Res. 2001;12:287-294.

7. Renvert S, Lessem J, Dahlen G, et al. Topical minocycline microspheres versus topical chlorhexidine gel as an adjunct to mechanical debridement of incipient peri-implant infections: A randomized clinical trial. J Clin Periodontol. 2006; 33:362-369.

8. Heitz-Mayfield LJA, Salvi GE, Mombelli A, et al. Anti-infective surgical therapy of periimplantitis. A 12-month prospective clinical study. Clin Oral Implants Res. 2012;23: 205-210.

9. Papadopoulos CA, Vouros I, Menexes $G$, et al. The utilization of a diode laser in the surgical treatment of peri-implantitis. A randomized clinical trial. Clin Oral Investig. 2015;19:1851-1860.

10. Hallstrom $H$, Persson GR, Lindgren S, et al. Open flap debridement of peri-implantitis with or without adjunctive systemic antibiotics: A randomized clinical trial. J Clin Periodontol. 2017;44: 1285-1293.

11. Jepsen K, Jepsen S, Laine ML, et al. Reconstruction of peri-implant osseous defects: A multicenter randomized trial. J Dent Res. 2016;95:58-66.

12. Roccuzzo M, Gaudioso L, Lungo $M$, et al. Surgical therapy of single periimplantitis intrabony defects, by means of deproteinized bovine bone mineral with 10\% collagen. J Clin Periodontol. 2016; 43:311-318.

13. Schwarz F, John G, Schmucker A, et al. Combined surgical therapy of advanced peri-implantitis evaluating two methods of surface decontamination: A 7-year follow-up observation. J Clin Periodontol. 2017;44:337-342.

14. Khoury F, Buchmann R. Surgical therapy of peri-implant disease: A 3-year follow-up study of cases treated with 3 different techniques of bone regeneration. J Periodontol. 2001;72:14981508.

15. de Waal YC, Raghoebar GM, Huddleston Slater JJ, et al. Implant decontamination during surgical periimplantitis treatment: A randomized, double-blind, placebo-controlled trial. J Clin Periodontol. 2013;40:186-195.

16. Romeo E, Lops D, Chiapasco M, et al. Therapy of peri-implantitis with resective surgery. A 3-year clinical trial on rough screw-shaped oral implants. Part II: Radiographic outcome. Clin Oral Implants Res. 2007;18:179-187.

17. Romeo E, Ghisolfi M, Murgolo N et al. Therapy of peri-implantitis with resective surgery. A 3-year clinical trial on rough screw-shaped oral implants. Part I: Clinical 
outcome. Clin Oral Implants Res. 2005;16: 9-18.

18. Carcuac O, Derks J, Abrahamsson I, et al. Surgical treatment of peri-implantitis: 3-year results from a randomized controlled clinical trial. J Clin Periodontol. 2017;44: 1294-1303.

19. Clem D, Rosen P, Cochran D, et al. Peri-implant mucositis and peri-implantitis: A current understanding of their diagnoses and clinical implications. J Periodontol. 2013;84:436-443.

20. Higgins JP, Altman DG, Gotzsche PC, et al. The Cochrane collaboration's tool for assessing risk of bias in randomised trials. BMJ. 2011;343:d5928.

21. Schulz KF, Altman DG, Moher D. CONSORT 2010 statement: Updated guidelines for reporting parallel group randomised trials. J Clin Epidemiol. 2010; 63:834-840.

22. Carcuac O, Derks J, Charalampakis $\mathrm{G}$, et al. Adjunctive systemic and local antimicrobial therapy in the surgical treatment of peri-implantitis: A randomized controlled clinical trial. J Dent Res. 2016; 95:50-57.

23. de Waal YC, Raghoebar GM, Meijer $\mathrm{HJ}$, et al. Implant decontamination with $2 \%$ chlorhexidine during surgical periimplantitis treatment: A randomized, double-blind, controlled trial. Clin Oral Implants Res. 2015;26:1015-1023.

24. Koldsland OC, Wohlfahrt JC, Aass AM. Surgical treatment of periimplantitis: Prognostic indicators of short-term results. J Clin Periodontol. 2018;45:100-113.
25. Raghavendra M, Koregol A, Bhola S. Photodynamic therapy: A targeted therapy in periodontics. Aust Dent J. 2009;54(suppl 1):S102-S109.

26. Heitz-Mayfield LJ, Mombelli A. The therapy of peri-implantitis: A systematic review. Int J Oral Maxillofac Implants. 2014;29(suppl):325-345.

27. Sahm N, Becker J, Santel T, et al. Non-surgical treatment of peri-implantitis using an air-abrasive device or mechanical debridement and local application of chlorhexidine: A prospective, randomized, controlled clinical study. J Clin Periodontol. 2011;38:872-878.

28. Serino G, Turri A, Lang NP. Maintenance therapy in patients following the surgical treatment of peri-implantitis: A 5-year follow-up study. Clin Oral Implants Res. 2015;26:950-956. 